\title{
Tickets Sales Policy for Two-stage Fares Airline considering Unidirectional Substitution of Cabin and Swapping Applications
}

\author{
Wang Fengchao ${ }^{1}$, Mo Zhenxiu², Li Cui $^{3, \text { a }}$ \\ ${ }^{1,2,3}$ School of Management and Economics, University of Electronics Science and Technology of \\ China, Chengdu,610054 \\ alicui20051018@126.com
}

Keywords: revenue management; sales policy; unidirectional substitution; swapping applications

\begin{abstract}
Tickets sales policy of two routes for two-stage fares airline are studied under the following three conditions in this paper: neither unidirectional substitution of cabin nor swapping applications is considered, only unidirectional substitution of cabin is considered, both unidirectional substitution of cabin and swapping applications are considered. We formulate the corresponding decision model and derive the optimal tickets amount and expected revenue. It shows that compared with the condition that neither unidirectional substitution of cabin nor swapping applications is considered, when only unidirectional substitution of cabin is considered, the expected optimal tickets amount and expected revenue increase; compared with the condition that only unidirectional substitution of cabin is considered, when both unidirectional substitution of cabin and swapping applications are considered, the expected optimal tickets amount and expected revenue increase.
\end{abstract}

\section{Introduction}

As a new development pattern of modern economy, aviation economy is becoming one of the key points. At the same time, the competitions between airlines are increasingly fierce. In the face of opportunities and challenges, if the airlines want to win in the competition, they must enhance capacity management and optimize ticket sales policy. As important approaches of revenue management, seats management and swapping applications are paid much attention and investigated by research institutions and enterprises. However, neither the theoretical study nor practical application has reached expectation. If the policies of seats management and swapping applications are combined effectively, it would create a larger space for the development of capacity management of airlines and be highly beneficial to increase customer satisfaction and revenue. Therefore, the study on tickets sales policy for two-stage fares airline considering unidirectional substitution and swapping applications has important practical significance.

Currently, the researches on airfare sales made by domestic and foreign scholars can be divided into two aspects which are static models and dynamic models. In the study of static models, Robinson (1995) ${ }^{[1]}$ addresses the discount bookings question and indicates the optimal policies. Talluri and Van Ryzin (2004) ${ }^{[2]}$ analyze a single-leg reserve management problem by constructing a choice model and obtain the optimal policy. Lohatepanont and Barnhart (2004) ${ }^{[3]}$ present integrated models and analyze the interactive influence between customer demand and airlines supply. Kunnumkal and Talluri (2012) ${ }^{[4]}$ consider the airfare sales problem and use a two-period stochastic linear program to make policies on seat inventory management and overbooking. Although the literatures above research on the tickets sales policy based on unfixed supply capacity, they do not integrate seats management with swapping applications effectively.

In the study of dynamic models, Liang $(1999)^{[5]}$ researches the yield management problem by constructing an expected revenue model. Zhao and Zheng $(2001)^{[6]}$ construct a two-class dynamic seats allocation model and derive structural properties of the optimal threshold policy. Levin etc. $(2009)^{[7]}$ present a dynamic pricing model for oligopolistic firms and obtain the optimal equilibrium solution. Chen etc. (2010) ${ }^{[8]}$ build a two-flight model to solve the optimal seat allocation problem and use a numerical example to illustrate the derivation of the optimal booking policies. The above 
literatures study the ticket sales policy mainly based on seats management and are less involved swapping applications, also lack of organic combination of both.

In summary, in the aspect of theoretical study, current researches mainly focus on unidirectional substitution or swapping applications separately. Therefore, with an organic integration of unidirectional substitution of cabin and swapping applications, the tickets sales policy based on a flexible capacity of supply is studied in this paper, which does not only make up the deficiency of current researches but also provide guidance for management practice of airline.

\section{Model descriptions and assumption}

Consider an airline with two routes which are route 1 and route 2 . The company previously arranges the big plane with seats $Q_{l}$ to fly route 1 and small plane with seats $Q_{s}$ to fly route $2\left(Q_{l}>Q_{s}\right)$. Seats in the big plane and the small plane are divided into first class and economy class, and of the total seats the ratio of the first class is $\alpha \cdot p_{i j}$ represents the price of class $j$ for route $i$; $D_{i j}$ represents the demand of class $j$ for route $i ; F_{i j}$ (.)and $f_{i j}$ (.) are the cumulative distribution function and probability density function of $D_{i j}$ respectively, $i=1,2, j=1,2$ ( $j=1$ represents the first class; $j=2$ represents the economy class). The ticket price $p_{i j}$ of route $i$ is an exogenous variable, and $p_{i 1}>p_{i 2}$.

Under the three conditions of neither unidirectional substitution of cabin nor swapping applications is considered, only unidirectional substitution is considered, both unidirectional substitution and swapping applications are considered, the optimal ticket policies to maximize expected revenue are investigated and the change of the optimal ticket amount and expected revenue are analyzed respectively. $T_{i j}, T_{i j}^{\prime}, T_{i j}^{\prime \prime}$ represent the ticket amount of class $j$ for route $i ; \pi_{i}, \pi_{i}^{\prime}, \pi_{i}^{\prime \prime}$ represent the revenue of route $i$ respectively.

Assumptions: (1) Service levels on both aircrafts are the same, which means passengers can accept the arrangement of swapping applications. (2) The demand of the first class and economy class for both planes is mutually independent. (3) The company will not swap aircrafts if passengers on the big plane would be denied after swapping. (4) The cost of swapping applications is ignored. (5) There are no overbooking and no-show for route 1 and 2.

\section{Models and solutions}

\section{Tickets sales policy without considering unidirectional substitution or swapping applications}

From actual investigation, we find that the airlines dynamically sell tickets based on customer demand. Thus, the airline can't adjust its carrying capacity without considering unidirectional substitution of cabin or swapping applications.

Proposition 1 without considering unidirectional substitution of cabin or swapping applications, there exist a unique optimal ticket amount for both route 1 and route 2 . The optimal ticket amount for route 1 is

$$
\left(T_{11}^{*}, T_{12}^{*}\right)=\left\{\begin{array}{ll}
\left(\alpha Q_{l},(1-\alpha) Q_{l}\right) & D_{11} \geq \alpha Q_{l}, D_{12} \geq(1-\alpha) Q_{l} \\
\left(\alpha Q_{l}, D_{12}\right) & D_{11} \geq \alpha Q_{l}, D_{12}<(1-\alpha) Q_{l} \\
\left(D_{11},(1-\alpha) Q_{l}\right) & D_{11}<\alpha Q_{l}, D_{12} \geq(1-\alpha) Q_{l} \\
\left(D_{11}, D_{12}\right) & D_{11}<\alpha Q_{l}, D_{12}<(1-\alpha) Q_{l}
\end{array} .\right.
$$

And the optimal ticket amount for route 2 is

$$
\left(T_{21}^{*}, T_{22}^{*}\right)=\left\{\begin{array}{ll}
\left(\alpha Q_{s},(1-\alpha) Q_{s}\right) & D_{21} \geq \alpha Q_{l}, D_{22} \geq(1-\alpha) Q_{l} \\
\left(\alpha Q_{s}, D_{22}\right) & D_{21} \geq \alpha Q_{l}, D_{22}<(1-\alpha) Q_{l} \\
\left(D_{21},(1-\alpha) Q_{s}\right) & D_{21}<\alpha Q_{l}, D_{22} \geq(1-\alpha) Q_{l} \\
\left(D_{21}, D_{22}\right) & D_{21}<\alpha Q_{l}, D_{22}<(1-\alpha) Q_{l}
\end{array} .\right.
$$

Proof: without considering unidirectional substitution of cabin or swapping applications, airlines 
try to meet the demand under the limit of carrying capacity. When $D_{11} \geq \alpha Q_{l}, D_{12} \geq(1-\alpha) Q_{l}$, the optimal ticket amount $\left(T_{11}^{*}, T_{12}^{*}\right)$ is $\left(\alpha Q_{l},(1-\alpha) Q_{l}\right)$.When $D_{11} \geq \alpha Q_{l}, D_{12}<(1-\alpha) Q_{l},\left(T_{11}^{*}, T_{12}^{*}\right)=\left(\alpha Q_{l}, D_{12}\right)$. When $D_{11}<\alpha Q_{l}, D_{12} \geq(1-\alpha) Q_{l},\left(T_{11}^{*}, T_{12}^{*}\right)=\left(D_{11},(1-\alpha) Q_{l}\right)$. When $D_{11}<\alpha Q_{l}, D_{12}<(1-\alpha) Q_{l},\left(T_{11}^{*}, T_{12}^{*}\right)=\left(D_{11}, D_{12}\right)$. Thus we can get the optimal ticket amount of route 1 . Similarly, we can get the optimal ticket amount of route 2 .

From proposition 1, the optimal ticket amount relates to customer demand and seats of aircrafts. Based on the demand and carrying capacity of aircrafts, the airline could select the optimal ticketing policy to achieve the maximization of the expected revenue.

\section{tickets sales policy with only considering unidirectional substitution of cabin}

Airlines could encounter such a situation that there are empty seats of the first class and the demand of economy class couldn't be met for the same route. In that case, the unidirectional substitution of cabin can not only meet demand of customer, but also improve the carrying capacity.

Proposition 2 with only considering unidirectional substitution of cabin, there exist a unique optimal ticket amount for both route 1 and route 2 . The optimal ticket amount for route 1 is

$$
\left(T_{11}^{\prime *}, T_{12}^{\prime *}\right)=\left\{\begin{array}{ll}
\left(\alpha Q_{l},(1-\alpha) Q_{l}\right) & D_{11} \geq \alpha Q_{l}, D_{12} \geq(1-\alpha) Q_{l} \\
\left(\alpha Q_{l}, D_{12}\right) & D_{11} \geq \alpha Q_{l}, D_{12}<(1-\alpha) Q_{l} \\
\left(D_{11}, Q_{l}-D_{11}\right) & D_{11}<\alpha Q_{l}, D_{11}+D_{12} \geq Q_{l} \\
\left(D_{11}, D_{12}\right) & D_{11}<\alpha Q_{l}, D_{11}+D_{12}<Q_{l}
\end{array} .\right.
$$

And the optimal ticket amount for route 2 is

$$
\left(T_{21}^{\prime *}, T_{22}^{\prime *}\right)=\left\{\begin{array}{ll}
\left(\alpha Q_{s},(1-\alpha) Q_{s}\right) & D_{21} \geq \alpha Q_{s}, D_{22} \geq(1-\alpha) Q_{s} \\
\left.\alpha Q_{s}, D_{22}\right) & D_{21} \geq \alpha Q_{s}, D_{22}<(1-\alpha) Q_{s} \\
\left.D_{21}, Q_{s}-D_{21}\right) & D_{21}<\alpha Q_{s}, D_{21}+D_{22} \geq Q_{s} \\
\left(D_{21}, D_{22}\right) & D_{21}<\alpha Q_{s}, D_{21}+D_{22}<Q_{s}
\end{array} .\right.
$$

Proof: with only considering unidirectional substitution of cabin, the big plane flies route 1 and the small plane flies route 2. When $D_{11}<\alpha Q_{l}, D_{12} \geq(1-\alpha) Q_{l}$, unidirectional substitution is conducted because first class has spare seats and economy class has extra passengers. The optimal ticket amount is $\left(D_{11}, D_{12}\right)$. When $D_{11} \geq \alpha Q_{l}, D_{12} \geq(1-\alpha) Q_{l}$ or $D_{11} \geq \alpha Q_{l}, D_{12}<(1-\alpha) Q_{l}$, first class has no spare seat. When $D_{11}<\alpha Q_{l}, D_{12}<(1-\alpha) Q_{l}$, economy class has no extra passenger. In all those three conditions, there is no need for unidirectional substitution and the optimal ticket amount is the same as what shows in proposition 1.Thus we can get the optimal ticket amount of route 1 . Similarly, we can get the optimal ticket amount of route 2 .

From proposition 2, the optimal ticket amount relates to the demand of customer and seats of aircrafts. When making the tickets sales decision, if the demand of first class is less than seats and the demand of economy class exceeds the seats, the airline could increase the ticket sales amount of economy class and meet the demand of passengers of economy class by substituting first class to economy class, thereby improving the utilization of resource.

Proposition 3 the optimal ticket amount of first class for route 1 and route 2 are both constant, while the expected optimal ticket amount of economy class and the expected revenue of the airline both increase with only considering unidirectional substitution of cabin.

Proof: from equation (1) and (3), we can get $T_{11}^{*}=T_{11}^{*}$, and compute the expected revenue of each route. Then $E\left(T_{12}^{\prime *}\right)-E\left(T_{12}^{*}\right)=\int_{0}^{\alpha Q_{l}} F_{11}(x) d x-\int_{0}^{\alpha Q_{l}} \int_{(1-\alpha) Q_{l}}^{Q_{l}-x} f_{11}(x) F_{12}(y) d y d x \geq 0, E\left(T_{12}^{\prime *}\right) \geq E\left(T_{12}^{*}\right)$. Similarly, we can demonstrate the change of the optimal ticket amount for route 2 .

From proposition 1 and 2, the change of the expected revenue for route 1 is

$$
\Delta E\left(\pi_{1}\right)=E\left(\pi_{1}^{\prime}\right)-E\left(\pi_{1}\right)=p_{12}\left[\int_{0}^{\alpha Q_{l}} F_{11}(x) d x-\int_{0}^{\alpha Q_{l}} \int_{(1-\alpha) Q_{l}}^{Q_{l}-x} f_{11}(x) F_{12}(y) d y d x\right] \geq 0
$$

Similarly, for route 2, the change of the expected revenue is $\Delta E\left(\pi_{2}\right)=E\left(\pi_{2}^{\prime}\right)-E\left(\pi_{2}\right) \geq 0$. Hence the change of expected revenue of the airline is $\Delta E(\pi)=\Delta E\left(\pi_{1}\right)+\Delta E\left(\pi_{2}\right) \geq 0$.

Proposition 3 shows that unidirectional substitution of cabin is beneficial for both passengers and 
airlines. For the passengers, unidirectional substitution of cabin has no effect on the boarding of first class passengers and can increase the boarding probability of economy class passengers. That's, it can better meet the demand of economy class passengers, enable them to enjoy the resources and services of first-class with the price of economy class and further improve their satisfaction. For the airlines, when the demand of first class is less than seats and the demand of economy class exceeds the seats, unidirectional substitution of cabin can make more economy class passengers boarding, which not only improves the utilization of resources effectively, but also increases the revenue of company by increasing the ticket amount.

Therefore, based on the actual situation, implementing unidirectional substitution of cabin at the right time is an effective mean of revenue management.

\section{considering unidirectional substitution of cabin and swapping applications}

Through unidirectional substitution of cabin, the contradiction between demand and supply in the routes can be solved in some extent, but the allocation of cabin between routes is still imbalance. That's, there are empty seats of one route while the customer demand of another can't be met. The Airline can both consider unidirectional substitution of cabin and swapping applications to improve the capacity of routes.

Proposition 4 there exist a unique optimal ticket amount for route 1 and route 2 with considering unidirectional substitution of aircraft cabin and swapping applications.

Proof: when considering swapping applications and unidirectional substitution of cabin at the same time, only swapping applications would be considered if unidirectional substitution is not carried out; unidirectional substitution would be considered after swapping applications if the airline swaps the aircraft.

(1) When $D_{11}>\alpha Q_{s}, D_{11}+D_{12}>Q_{s}$ or $D_{21} \leq \alpha Q_{s}, D_{21}+D_{22} \leq Q_{s}$, there's no need for swapping applications. For route 1 , if $D_{11}<\alpha Q_{l}$ and $D_{12}>(1-\alpha) Q_{l}$, substitution is conducted; if $D_{11} \geq \alpha Q_{l}$ or $D_{12} \leq(1-\alpha) Q_{l}$, there's no need for substitution. For route 2, if $D_{21}<\alpha Q_{s}$ and $D_{22}>(1-\alpha) Q_{s}$, substitution is conducted; if $D_{21} \geq \alpha Q_{s}$ or $D_{22} \leq(1-\alpha) Q_{s}$, there's no need for substitution.

(2) When $D_{11} \leq \alpha Q_{s}, D_{11}+D_{12} \leq Q_{s}, D_{21}>\alpha Q_{s}$ or $D_{21}+D_{22}>Q_{s}$, swapping applications is conducted. For route 1 , if $D_{11}<\alpha Q_{s}$ and $D_{12}>(1-\alpha) Q_{s}$, substitution is conducted; if $D_{11} \leq \alpha Q_{s}$ or $D_{12} \leq(1-\alpha) Q_{s}$, there's no need for substitution. For route 2, if $D_{21}<\alpha Q_{l}$ and $D_{22}>(1-\alpha) Q_{l}$, substitution is conducted; if $D_{21} \geq \alpha Q_{l}$ or $D_{22} \leq(1-\alpha) Q_{l}$, there's no need for substitution. Thus we can get the ticket amount of every route under different conditions.

From proposition 4, the airline selects the ticketing strategy according to the demand of customer and improves the carrying capacity of route 2 by swapping the aircrafts in certain circumstances to make better use of resources and meet the demand of customers. Next, the change of optimal ticket amount of first class and economy class for route 1 and route 2 is explored.

Proposition 5 compared with only considering unidirectional substitution, optimal ticket amount of first class and economy class for route 1 are constant while that of route 2 increase under the consideration of unidirectional substitution and swapping applications.

Proof: from proposition 2 and 4, compared with only considering unidirectional substitution, optimal ticket amount of first class and economy class for route 1 are constant under the consideration of unidirectional substitution and swapping applications.

Then check the change of optimal ticket amount of first class for route 2. From proposition 2 and proposition 4, we know $E\left(T_{21}^{\prime \prime \prime}\right)-E\left(T_{21}^{\prime *}\right)=\left[\alpha Q_{l}-\alpha Q_{s}-\int_{\alpha Q_{s}}^{\alpha Q_{l}} F_{21}(t) d t\right] \int_{0}^{\alpha Q_{s}} f_{11}(x) F_{12}\left(Q_{s}-x\right) d x \geq 0$.Therefore, under the consideration of unidirectional substitution and swapping applications, the optimal ticket amount of first class for route 2 increases. Similarly, we can get

$$
\begin{aligned}
& E\left(T_{22}^{\prime \prime *}\right)-E\left(T_{22}^{\prime \prime}\right) \geq \int_{0}^{\alpha Q_{s}} f_{11}(x) F_{12}\left(Q_{s}-x\right) d x\left[\int_{0}^{\alpha Q_{s}} f_{21}(t) \int_{(1-\alpha) Q_{s}}^{Q_{s}-t} F_{22}(s) d s d t-\int_{0}^{\alpha Q_{s}} F_{21}(t) d t+\int_{0}^{\alpha Q_{l}} F_{21}(t) d t\right] \\
& -\int_{0}^{\alpha Q_{l}} f_{21}(t) \int_{(1-\alpha) Q_{l}}^{Q_{l}-t} F_{22}(s) d s d t \geq 0
\end{aligned}
$$


Therefore, the optimal ticket amount of economy class for route 2 increases.

The above proposition shows that as the large aircraft is the default model of route 1 , the airline can't resolve the contradiction of mismatch between supply and demand by swapping applications, and only could meet the demand of customers with unidirectional substitution when the demand of route 1 exceeds the capacity of large aircraft. Therefore the optimal ticket amount of route 1 with only considering unidirectional substitution is the same as that of route 1 under the consideration of unidirectional substitution and swapping applications.

When the demand of route 1 exceeds the capacity of small aircraft, the airline can meet the demand of route 2 by swapping applications in some cases and further conduct unidirectional substitution of cabin. Thus, the expected optimal ticket amount of first class and economy class for route 2 both increase.

Based on the above analysis, when unidirectional substitution and swapping applications are both considered, the airline is more at ease in the use of capacity management policies and it is much more convenient for the boarding of passengers. From the aspect of airlines, unidirectional substitution from first class to economy class can realize the maximum utilization of cabin resources and swapping applications reallocates the cabin resources between the two routes, thereby increasing the flexible of capacity and enhancing the utilization of cabin resources better. From the aspect of customers, under the condition that the service levels of the two aircrafts are the same, unidirectional substitution and swapping applications not only guarantee the boarding and quality service of first class, but also make more economy class passengers boarding. Their demand can be better met and they feel more satisfied.

Proposition 6 compared with only considering unidirectional substitution, the expected revenue of route 1 is constant while that of route 2 and the airline both increase under the consideration of unidirectional substitution and swapping applications.

Proof: under the consideration of unidirectional substitution and swapping applications, the revenue of route 1 is $\pi_{1}^{\prime \prime}=\left(T_{11}^{\prime \prime *}, T_{12}^{\prime \prime *}\right) \cdot\left(p_{11}, p_{12}\right)^{T}$. Under the consideration of unidirectional substitution, the revenue of route 1 is $\pi_{1}^{\prime}=\left(T_{11}^{\prime *}, T_{12}^{\prime *}\right) \cdot\left(p_{11}, p_{12}\right)^{T}$. From proposition 5, $\left(T_{11}^{\prime \prime *}, T_{12}^{\prime \prime *}\right)=\left(T_{11}^{\prime *}, T_{12}^{\prime *}\right)$, then $\pi_{1}^{\prime \prime}=\pi_{1}^{\prime}$. Hence the revenue of route 1 is constant.

Under the consideration of unidirectional substitution and swapping applications, the expected revenue of route 2 is $E\left(\pi_{2}^{\prime \prime}\right)=p_{21} E\left(T_{21}^{\prime \prime \prime}\right)+p_{22} E\left(T_{22}^{\prime \prime *}\right)$. Under the consideration of unidirectional substitution, the expected revenue of route 2 is $E\left(\pi_{2}^{\prime}\right)=p_{21} E\left(T_{21}^{\prime *}\right)+p_{22} E\left(T_{22}^{\prime *}\right)$. From proposition 5, we know $E\left(T_{21}^{\prime \prime \prime}\right) \geq E\left(T_{21}^{\prime *}\right) E\left(T_{22}^{\prime \prime *}\right) \geq E\left(T_{22}^{\prime *}\right)$. Hence $E\left(\pi_{2}^{\prime \prime}\right) \geq E\left(\pi_{2}^{\prime}\right)$, the expected revenue of route 2 and the airline both increase.

Proposition 6 shows that the airline should combine those two revenue management methods to better meet the demand of customers and improve the expected revenue.

\section{Conclusions}

Based on unfixed supply capacity of the airline, tickets sales policy for two-stage fares airline are studied in this paper. The decision models are formulated under the three conditions: neither unidirectional substitution of cabin nor swapping applications is considered, only unidirectional substitution of cabin is considered, both unidirectional substitution of cabin and swapping applications are considered. Then the optimal ticket amount and the expected revenue are derived respectively. Comparative analysis of the optimal solutions is given afterwards and we obtain the conclusions as follows: (1)there exist unique optimal tickets amount with different demands under those three conditions; (2)compared with the condition that neither unidirectional substitution of cabin nor swapping applications is considered, the optimal ticket amount of first class for both routes are constant, while the expected optimal ticket amount of economy class and the expected revenue of the airline both increase with only considering unidirectional substitution of cabin. (3)compared with only considering unidirectional substitution, optimal ticket amount of first class and economy class for route 1 are constant while that of route 2 and the expected revenue of the 
airline both increase under the consideration of unidirectional substitution and swapping applications.

In this paper, the tickets sales policy for two-stage fares airline is investigated without considering overbooking or no-show. Therefore, based on the unfixed supply capacity, the further study can be conducted on the tickets sales policy for two-stage fares airline with considering overbooking and no-show.

\section{References}

[1] Lawrence W. Robinson, Optimal and approximate control policies for airline booking with sequential nonmonotonic fare classes, J. Operations Research. 43(2) (1995)252-263.

[2] Talluri K., van Ryzin G, Revenue management under a general discrete choice model of consumer behavior, J. Management Science. 50(2004)15-33.

[3] Lohatepanont M., C. Barnhart, Airline schedule planning: integrated models and algorithms for schedule design and fleet assignment, J. Transportation Science. 38(1) (2004)19-32.

[4] Kunnumkal S., TalluriK A, Randomized linear programming method for network revenue management with product-specific no-Shows, J. Transportation Science. 46(1) (2012)90-108.

[5] Liang Y, Solution to the continuous time dynamic yield management model, J. Transportation Science. 33(1999)117-123.

[6] Zhao W., Zheng Y. S, A dynamic model for airline seat allocation with passenger diversion and no-show, J. Transportation Science. 35(2001)80-98.

[7] Levin Y., McGill J. I., Nediak M, Dynamic pricing in the presence of strategic consumers and oligopolistic competition, J. Management Science. 55(1) (2009)32-46.

[8] Chen S., Gallego G., Michael Z.F. Li, Lin B, Optimal seat allocation for two-flight problems with a flexible demand segment, J. European Journal of Operational Research. 201(3) (2010)897-908. 